\title{
Breastfeeding and colostrum feeding practices adopted by mothers of infants (6-30 Months)
}

\author{
N. Panwar and M. Mathur
}

Infant feeding practices is prevailing universally and considered important determinants for development of infants. The feeding practices develop immunity in the infants to fight against diseases in the later phase of their life. In the present study, feeding practices include both breast feeding and colostrum feeding practices. A study was conducted in Jodhpur, Rajasthan to assess the feeding practices of infants among the working - non working mothers of rural - urban areas. The purposive sampling technique was used to select 240 infants, 120 infants belonged to working mothers (60 infants from each rural - urban area) and 120 infants of non-working mothers (60 infants from each rural and urban area). The data was collected by interview method by using semi structure and standarizised questionnaire based on the guidelines of World Health Organization (WHO) on breastfeeding. The evaluation criteria was also framed and standardization was done along with the formation of the tool for the study. The responses were categorized under good and poor ranking. The result shows a significant association between the feeding practices - breastfeeding and colostrum feeding practices among mothers of rural- urban areas. Majority $(96.7 \%)$ of mothers exclusively breastfeed their infants. The non working mothers of rural areas breastfeed their infants for more than 18 months. Insufficient milk secretion was the most common reason for delay of breastfeeding among the working mothers of rural areas (93.3\%). The working mothers of rural areas, who fed their infants according to the 'schedule', were rated good whereas poor rating was given to non working mother of rural areas who had breastfeed their infants 'on demand'. Lack of knowledge and superstitions prevailing among the non working mothers $(1.3 \%)$ of rural areas which was one of the reasons for onset of next pregnancy which affects the breast feeding practices. Discard of colostrum was found among the non working mothers of rural areas i.e. maximum of 43.3\%. Based on the result, it can be concluded that exclusive feeding practice among the mothers of rural-urban areas was upto the standard. These findings highlighted that mothers should be given proper knowledge of feeding practices both in rural and urban areas during the pregnancy itself.

Key Words : Working non-working mothers, Rural-urban areas, Feeding practices, Exclusive breast feeding, Colostrum, Pre lacteal feed

How to cite this article : Panwar, N. and Mathur, M. (2017). Breastfeeding and colostrum feeding practices adopted by mothers of infants (6-30 Months). Food Sci. Res. J., 8(2): 290-297, DOI : 10.15740/HAS/FSRJ/8.2/290-297.

M. MATHUR, Department of Home Science, Jai Narain Vyas University, JODHPUR (RAJASTHAN) INDIA

Email : mmathur26@yahoo.co.in 